Esta obra está bajo una Licencia Creative Commons

Atribución-NoComercial-Compartirlgual 4.0 Internacional

La Tutoría pedagógica y el oficio de estudiante: el desarrollo de hábitos

y prácticas en el proceso de adaptación a la universidad.

Laura Marín

Trayectorias Universitarias, 7 (13), e079, 2021

ISSN 2469-0090 | https://doi.org/10.24215/24690090e079

https://revistas.unlp.edu.ar/TrayectoriasUniversitarias

Universidad Nacional de La Plata

La Plata | Buenos Aires | Argentina

\title{
La Tutoría pedagógica y el oficio de estudiante: el desarrollo de hábitos y prácticas en el proceso de adaptación a la universidad.
}

The pedagogic tutorship and the learner's experience: the development of habits and practice in the process of adapting to university

Laura Marín

https://orcid.org/0000-0002-9134-5690

Imarin@unlam.edu.ar

Universidad Nacional de La Matanza |

Argentina

\section{RESUMEN}

El artículo tiene como propósito presentar los resultados de una investigación sobre la incidencia de la Tutoría pedagógica del Departamento de Derecho y Ciencia política de UNLaM en los estudiantes que cursan la carrera de Abogacía. Para ello en primer lugar, se presenta el marco conceptual y luego los aspectos metodológicos. Seguidamente se analizan fragmentos extraídos de entrevistas realizadas a los estudiantes tutorados los cuales permiten avanzar en la identificación de cuatro aspectos vinculados al desarrollo de hábitos y prácticas en el oficio de estudiar y la adaptación a la universidad: lo actitudinal, ligado a temores y desorientación inicial; la autonomía, vivida como una paradoja entre la libertad y la autodisciplina; los tiempos y el calendario, vinculados a la organización como factor clave y los hábitos de estudio desarrollados a través de métodos y estrategias para abordar la tarea. Por último, y a modo de cierre, se plantean algunas conclusiones a partir del análisis de los datos.

PALABRAS CLAVE

tutoría,

estudiante,

adaptación, universidad 
KEY WORDS

tutorship,

student,

adaptation,

university.

\section{ABSTRACT}

This article aims at presenting the results of an investigation about the impact of Pedagogic Tutorship from the Law and Political Sciences Department at UNLaM on the students attending the Law course of studies. Thus, firstly, the conceptual framework is presented and then, the methodological aspects. Secondly, extracts from interviews to tutored students are analyzed. These extracts allow us to identify four aspects linked to the development of habits and learner's experience practices and their adaptation to university: the attitudinal aspect, connected to their fears and initial confusion; the autonomy, seen as freedom in direct contradiction to self-discipline; the schedule, linked to organization as a key point and the studying habits developed through methods and strategies to deal with their tasks. Finally, some conclusions are drawn from the analysis of data. 


\section{INTRODUCCIÓN}

En estos tiempos de cambio e innovación en la educación superior, la tutoría universitaria se presenta como uno de los tópicos protagonistas en relación con la formación integral de los estudiantes y su rol como eje articulador de recursos y voluntades en el acompañamiento de las trayectorias educativas.

Desde sus comienzos, se constituye en un método de aprendizaje integrado a los planes de estudio que permite el seguimiento, la supervisión y evaluación personalizada de la tarea.

La tutoría es un componente inherente a la formación académica pues favorece la adaptación a la universidad, el aprendizaje y rendimiento académico, la orientación curricular y profesional. (Rodriguez Espinar, 2004)

En el presente trabajo analizaremos la incidencia de la tutoría en la formación del estudiante y su afiliación a la nueva organización de educación superior a partir de la investigación que se lleva a cabo en la carrera de Abogacía denominada "La acción tutorial en la universidad: un estudio sobre la tutoría pedagógica como estrategia de acompañamiento a las trayectorias académicas de los estudiantes de la carrera de Abogacía del Departamento de Derecho y Ciencia Política en UNLaM"

\section{MARCO CONCEPTUAL}

Coulon (2005) considera la entrada a la universidad como un tránsito o pasaje de un estatus social a otro, de una cultura a otra. Desde el sentido otorgado por la etnografía como estudio directo de personas o grupos durante un cierto período para conocer su comportamiento social (Guiddens, 2001), el pasaje exige una iniciación debido a que lo primero que el estudiante está obligado a hacer es aprender su oficio de estudiante.

El proceso de ingreso se daría en tres tiempos que son el tiempo de la alienación o entrada a un universo desconocido que rompe con el mun- 
do anterior; el tiempo del aprendizaje o movilización de energías, definición de estrategias, adaptación progresiva; y el tiempo de la afiliación o de relativo dominio de las reglas institucionales. En tanto el pasaje sea satisfactorio el estudiante pasa de su condición de novato a la condición de aprendiz y de allí a la de miembro afiliado. En este sentido Coulon distingue dos tipos de afiliación: institucional e intelectual. La primera tiene que ver con el conocimiento de los modos de funcionamiento de la universidad, la organización administrativa y funcional, las normas que regulan su funcionamiento; la segunda tiene que ver con el dominio de las formas de trabajo intelectual que implica la comprensión de conceptualizaciones, categorizaciones, discursos y prácticas propias de la organización universitaria (Coulon, 2005).

Es decir, el desenvolvimiento de los procesos de afiliación no es necesariamente simultáneo. Es probable que el estudiante apruebe las situaciones organizativo académicas pero no se adapte a las exigencias de la esfera intelectual lo que explicaría la presencia de un grupo considerable de estudiantes visibles en lo administrativo pero no en lo pedagógico. El mundo de la universidad es nuevo para los estudiantes que deben comprender las complejidades de la institución, del plan de estudios, de las normas.

\section{El mundo de la universidad es nuevo para los estudiantes que deben comprender las complejidades de la institución, del plan de estudios, de las normas.}

La noción de afiliación que acuna Coulon (2005) parecería apropiada para distinguir el tipo de afiliación intelectual o cognitiva, es decir, la comprensión de lo que profesores e institución esperan del estudiante para considerar su condición de tal. Más allá de la integración, consiste en naturalizar prácticas y funcionamientos universitarios a fin de convertirse en un miembro competente de la comunidad académica. Desde el punto de vista intelectual sería saber identificar el trabajo no pedido explícitamente, saber reconocerlo y el momento en que cumplirlo. También saber comprender los códigos de trabajo intelectual contenidos en un conjunto de reglas muchas veces informales e implícitas. Es en este sentido que el acompañamiento pedagógico colabora en la tarea de identificar un trabajo intelectual en el hacer concreto.

De este modo la tutoría se transforma en un dispositivo que favorece y orienta los procesos educativos constituyéndose en un medio para el mejoramiento de las trayectorias educativas de los estudiantes con atención a sus necesidades y condiciones particulares (Camborius de Donini, 2011) 
En cuanto al ejercicio del oficio, la adquisición de la habilidad es sustancial. Habilidad se remonta a la raíz lingüística de poièin, que significa "hacer", en verdad se la define como "práctica entrenada" (Sennett, 2009:71). La asimilación constituye un proceso esencial para todas las habilidades debido a que transforma un comportamiento a tal punto que se convierte en rutina y no pensamos en él. Al aprender una habilidad el aprendiz desarrolla un complicado repertorio de procedimientos en donde se interrelacionan constantemente conocimiento tácito y reflexivo.

Trabajar la resistencia, explorar las diferentes maneras de llevar a cabo la práctica disponen al buen artesano a disminuir la tensión y aceptar la ambigüedad estableciendo una estrecha relación entre la solución y el descubrimiento de problemas, la técnica y la expresión, el juego y el trabajo

Es así como según Capelari (2018), la tutoría acompaña este proceso de transformación de alumno en estudiante orientando la construcción de habilidades requeridas para el acceso al conocimiento, participando en las prácticas académicas de la universidad y sosteniendo la motivación. En cierto sentido, el tutor depende de un estar y actuar pertinente en relación a los nuevos escenarios y funciones de los sistemas tutoriales.

La adaptación e integración a la comunidad universitaria es uno de los aspectos clave de la tutoría, especialmente al ingreso en la institución, creando un proceso de acompañamiento en el pasaje de la educación media a la educación universitaria.

\section{La adaptación e integración a la comunidad universitaria es uno de los aspectos clave de la tutoría, especialmente al in- greso en la institución, creando un proceso de acompaña- miento en el pasaje de la educación media a la educación universitaria.}

Aparecen nuevas formas de enseñar y aprender, modos de hacer, nuevos contenidos, compañeros y profesores que suponen una adaptación en la que se debe actuar desde la tutoría (Pérez Cursó, 2017)

\section{ASPECTOS METODOLÓGICOS}

Se realiza el seguimiento de una muestra de estudiantes de la carrera de Abogacía que ha participado del espacio de tutorías departamental y se trabaja sobre la reflexión de los ejes vinculados a la adaptación a la universidad y alfabetización académica.

A partir de la modalidad básica se lleva a cabo un estudio descrip- 
tivo que permite identificar nuevos temas de investigación a partir de la utilización de un método cuanti- cualitativo.

El diseño se conforma como un proceso interactivo que involucra diversos aspectos entre sus componentes enfatizando la comprensión de las estructuras significativas del contexto a fin de favorecer los procesos de entendimiento del ámbito de investigación.

En lo que se refiere a etapas de la investigación, primeramente seleccionamos la muestra, luego se procede a recoger la información mediante técnicas especificadas de entrevista y encuesta. Por último, nos dedicamos al análisis de la información y a la comunicación de los resultados. Sobre ellos nos ocupamos a continuación.

\section{PERCEPCIONES DE LOS ESTUDIANTES SOBRE LA INCIDENCIA DE LA TUTORÍA EN EL PROCESO DE FORMACIÓN DEL ESTUDIANTE Y SU ADAPTACIÓN A LA UNIVERSIDAD}

La tutoría departamental plantea varias modalidades de intervención comenzando en el primer año de la carrera con una Orientación General que trabaja sobre la transición escuela media- universidad, continuando con la tutoría de carrera en el acompañamiento de la trayectoria estudiantil académica y culminando con la tutoría de egreso en la transición universidad- mundo laboral. Las modalidades que se adoptan generan espacios de trabajo diferenciados. Sin embargo, es principalmente el área de Orientación General el que prioriza el acompañamiento pedagógico en cuanto a la formación del estudiante a los que hemos consultado sobre la incidencia de la tutoría en el desarrollo de hábitos y prácticas específicas realizados en la educación superior, para llevar a cabo satisfactoriamente el trayecto de carrera.

Los datos aportados por los estudiantes, permiten identificar algunas líneas referidas al desarrollo de hábitos y habilidades de estudio en el proceso de construcción del oficio de estudiante y adaptación académica a partir de su participación en las tutorías departamentales.

En principio a parece lo actitudinal ligado a temores, desorientación y fracturas con los esquemas de estudio empleados hasta el momento "...la tutoría me incentivó a querer leer porque sé que puedo obtener un buen resultado si aplico a lo que estoy haciendo, mejoró mi actitud". "Cuando iba a una clase ya mi predisposición no era la misma, sino que iba con la intención de tomar los apuntes, y al retirarme de la clase, sabía que venía el trabajo de investigación en mi casa"; "...te ayudan para que de una manera u otra puedas encontrar tu metodología o esquema para estudiar, en definitiva siempre lo que se transmite desde acá es que nosotros nos soltemos en cuanto a estudiar a leer y demás"

"Sí, yo era más introvertido. El venir a este espacio de tutoría me generó autoconfianza y me fui soltando, adquiriendo autonomía. El desapego se siente al comienzo. Ahora yo puedo ayudar a otros". 


\section{El cultivo de otras dimensiones del individuo como la con- fianza, la capacidad de resolución y la creatividad en la bús- queda de solución a problemas entre otras, complementan la formación superior contribuyendo a incrementar las posi- bilidades de éxito en el desempeño académico.}

El cultivo de otras dimensiones del individuo como la confianza, la capacidad de resolución y la creatividad en la búsqueda de solución a problemas entre otras, complementan la formación superior contribuyendo a incrementar las posibilidades de éxito en el desempeño académico. En este sentido la tutoría interviene en aspectos como la conducta, los procesos de pensamiento, la motivación, métodos y hábitos para el estudio. (Ariza Ordoñez, Ocampo Villegas, 2004)

En cuanto a la línea de autonomía los entrevistados viven una paradoja al respecto considerando por un lado la libertad e independencia de los estudios superiores que se contrapone a la imposición de una disciplina necesaria para desarrollar el curso de sus estudios. En este caso la autonomía que se va adquiriendo requiere de una madurez para la conversión de alumno a estudiante como lo evidencian las siguientes afirmaciones: "Me ayudó un montón en la autonomía igual todavía me falta, recién empiezo con los tiempos no son los mismos de una persona que estudia y no trabaja a una que tiene muchas actividades, cuesta un poquito más, cuesta también la concentración más que nada"

"En cuanto a ser estudiante universitario, yo no asumía ese rol. La tutoría modificó este error y a orientarme en la autonomía universitaria". "Me permitió crecer y valerme por mí misma. Presentarme al examen final y rendir satisfactoriamente". "Sí, me generó autodisciplina para hacer lo que debo hacer".

Se visibiliza la obligación de administrar la propia vida sin control social directo que se transforma en la aventura de conocerse a sí mismo. El papel de la información es uno de los elementos cruciales para el éxito de su logro.

Otra cuestión que aparece es la función organizadora de la tutoría en cuanto a horarios de los tiempos de estudio y calendario. Los estudiantes destacan la importancia del espacio en la toma de conciencia sobre el uso del tiempo como factor clave para mejorar el rendimiento y afianzar los conocimientos.

"Me ayudó muchísimo por ejemplo, como yo estoy muy ocupada desde la mañana muy temprano entonces traté de decir bueno este horario es para estudiary buscar el lugar también el lugar donde pueda estar concentrada porque ese era mi tema"; "Si porque o sea, yo ya sé que tengo que ir y sentarme a estudiar y quizá eso antes lo hacía en un ratito libre pero eran muy disparejos los tiempos y demás así que tomé más conciencia, me ayudó bastante"; "ahora cuando tengo que leer o sea para no distraer- 
me, o pongo un reloj y pienso en tanto tiempo voy a leer"... "el tema de los 40 minutos de concentración ... bueno me acomodo a estudiar dentro de un tiempo evitando distracciones, cosa de que por ahí digo, voy a lavar la ropa voy a hacer tal cosa en el medio y se me ocurren ideas de cosas que tengo que hacer, ahora las evito directamente pienso este tiempo es para estudiar, para la lectura";" vengo avanzando bastante en las lecturas de la materia que estoy cursando y vengo rindiendo bien los pequeños parciales de la materia con la que me quedé porque si hubiese seguido con las dos, no sabría a cuál apostar así que decidí por esta organización de los tiempos, me ayudó a elegir un camino de mayor seguridad" Es así como la función de planificación en la tutoría permite el diseño de itinerarios educativos personalizados según las características y necesidades propias de estudiante.

En este punto se produce la relación con otra cuestión que aparece que es la del desarrollo de los hábitos de estudio. Hernández Herrera, C (2012) sostiene que los hábitos de estudio se conceptualizan como los métodos y estrategias que suele utilizar un estudiante para hacer frente a una cantidad de contenidos de aprendizaje. El proceso de estudio es complejo por lo que requiere una adecuada planeación. "Sí, si bien yo tenía hábitos de estudio, la tutoría me enseñó a ser más previsor o precavido en mis estudios. Hay un salto abismal entre la escuela secundaria y la universidad". "No tenía hábitos incorporados para el estudio superior. Era desordenada. Me ayudó a organizar mis horarios, mantener una disciplina de lectura, organizar las materias que cursaba. Pude armonizar lo intelectual con lo personal y resté ansiedad." "El hábito de estudio principal es el de la lectura. El tema de ahondar en los temas y apegarme al programa y no apartarme de los contenidos. Acudir y comprender para qué está una bibliografía complementaria. Eso yo no lo comprendía, para qué estaba. Me di cuenta de que eso pudo hacer que además de los contenidos mínimos, pueda profundizar y no aprobar raspando"

\section{REFLEXIONES}

En el ámbito de la formación del estudiante y adaptación a la vida universitaria el espacio de tutoría cumple una función primordial. Mediante el acompañamiento realizado se favorece una mejor comprensión de los desafíos a los que se enfrenta el alumno en lo referente al ambiente universitario y se desarrollan competencias para el desenvolvimiento académico y logro de objetivos. 
En cuanto al aporte del espacio tutorial, las líneas definidas por los estudiantes en este sentido se relacionan con lo actitudinal, la autonomía, la organización de los tiempos de estudio y calendario y la formación de hábitos para la tarea.

Como indica Herrera Aponte (2006) la tutoría como proceso de acompañamiento es tarea de compromisos y responsabilidades compartidas, sin embargo en el trayecto, el espacio brinda la oportunidad del autoconocimiento, la reflexión sobre las propias prácticas, la organización cotidiana de la vida para centralizar el estudio en un lugar de relevancia que permita el logro de los propósitos planteados al ingreso en la universidad. La institución tiene la responsabilidad de posibilitar a los estudiantes un recorrido académico inclusivo orientado hacia el aprendizaje, que favorezca la retención con un estímulo y acompañamiento acordes a tamaña empresa.

\section{La institución tiene la responsabilidad de posibilitar a los estudiantes un recorrido académico inclusivo orientado ha- cia el aprendizaje, que favorezca la retención con un estímu- lo y acompañamiento acordes a tamaña empresa.}




\section{BIBLIOGRAFÍA}

Ariza Ordóez, Gladys Ibeth; Ocampo Villegas, Héctor Balmes (2005) El acompañamiento tutorial como estrategia de la formación personal y profesional: un estudio basado en la experiencia en una institución de educación superior Universitas Psychologica, vol. 4, núm. 1, enero-junio, 2005, pp. 31-41 Pontificia Universidad Javeriana Bogotá, Colombia. Disponible en: https://www.redalyc.org/pdf/647/64740104.pdf

Cambours de Donini, A., Iglesias, A., \& Muiños de Britos, S. M. (2011). La tutoría en la Universidad: una estrategia para la retención de los estudiantes. Congresos CLABES. Recuperado a partir de https://revistas.utp.ac.pa/index.php/ clabes/article/view/853

Capelari M. (2018) Reseñas del I Seminario Latinoamericano de políticas de Inclusión y Tutoría. Revista Tutorías en Educación Superior GITBA. ISSNe:2347-1069.Año4- №4- diciembre 2018. Disponible en: https://www.gitba. org/RevistaGitba/N04/RG-numero04.pdf

Coulon, A. (2005) Le Metier d'Etudiant. L'entre dans la vie universitaire. París. Económica.

Guiddens, A. (2001) Sociología. Madrid. Alianza

Hernández Herrera, Claudia Alejandra; Rodríguez Perego, Nicolás; Vargas Garza, Ángel Eduardo Los hábitos de estudio y motivación para el aprendizaje de los alumnos en tres carreras de ingeniería Revista de la Educación Superior, vol. XLI (3), núm. 163, julio-septiembre, 2012, pp. 67- 87 Asociación Nacional de Universidades e Instituciones de Educación Superior DF México. Disponible en: https:// www.redalyc.org/pdf/604/60425380005.pdf

Herrera Aponte (2006) El proceso de tutoría: Un ritual de esperanza. Educare, ISSN 1316-6212, ISSN-e 2244-7296, Vol. 10, №. 3, 2006, págs. 192-201. Disponible en: https://dialnet.unirioja.es/servlet/articulo?codigo=2551159

Pérez Cusó, F. J., González Lorente, C., González Morga, N., \& Martínez Juárez, M. (2017). Tutoría en la Universidad: un estudio de caso en la Facultad de Educación de la Universidad de Murcia. Educatio Siglo XXI, 35(2 Jul-Oct), 91-110. Disponible en: https://doi.org/10.6018/j/298531

Rodriguez Espinar (2008) Manual de Tutoría Universitaria. Octaedro- España

Sennett, (2009) El artesano. Barcelona. Anagrama

Rockwell, E. (2013). "El trabajo docente hoy: nuevas huellas, bardas y veredas". En XI Congreso Nacional de Investigación Educativa. Consejo Mexicano de Investigación Educativa. 